\title{
$\mathrm{PH} 10 \mathbf{0}_{\text {debate }}$
}

a debate Repositorios y redes sociales académicas para la transferencia del conocimiento abierto

| coordina Remedios Melero Melero

\section{Los repositorios institucionales en la era de las altmetrics}

\author{
Juan José Prieto-Gutiérrez | Universidad Complutense de Madrid \\ URL de la contribución <www.iaph.es/revistaph/index.php/revistaph/article/view/4665>
}

Los repositorios institucionales son plataformas de autoarchivo que recopilan, preservan y difunden la producción académica de una institución. El contenido es múltiple y variado, desde artículos de profesores e investigadores y trabajos de estudiantes, hasta colecciones especiales o documentos históricos de la Universidad.

La razón principal de los repositorios es aumentar la visibilidad de la producción propia. Pero dentro del actual contexto educativo, tanto la Universidad como los centros de investigación tienen una nueva misión: promover el acceso abierto, siendo a través de los repositorios institucionales como se garantiza parte del éxito del movimiento.

Cada vez más instituciones académicas reconocen el valor y beneficios de las herramientas de acceso a los documentos, pero desafortunadamente existen ciertos problemas técnicos y organizativos que se van solventando con el paso del tiempo.

Por ejemplo, la existencia de varios repositorios en una misma institución, en vez de ofrecer un punto de acceso único, la recolección de documentos por parte de bibliotecarios en vez de por los autores de los materiales, escasa formación en políticas de derechos de autor, mantenimiento y visualización de las plataformas, metadatos, etc.

Los repositorios institucionales están integrados en, al menos, cuatro contextos de aplicación (GONZÁLEZPÉREZ; RAMÍREZ-MONTOYA; GARCÍA-PEÑALVO, 2020):

$>$ Servicios tecnológicos, que garantizan la disponibilidad y seguridad de los recursos de información.

$>$ Arquitectura de la información y estándares de diseño.

$>$ Regulaciones institucionales y gubernamentales para

la difusión de acceso abierto.

> Métricas y criterios de evaluación.
Y es, en el punto cuarto, en relación con las métricas y más concretamente con las métricas alternativas, donde se va a centrar esta aportación.

Los datos, los números y los porcentajes son de gran importancia para el desarrollo diario de la sociedad y el área científica no se queda atrás.

El éxito de los repositorios estriba tanto en el acceso abierto de la documentación, como en el consumo o descarga de la misma. De nada serviría mantener un brillante repositorio institucional si el acceso es nulo. Por ello, en este sentido, las estadísticas de uso son esenciales, tanto para la institución académica como para el propio autor, al ofrecer la popularidad de un documento y su potencial de lectura y, en definitiva, el valor o influencia científica y académica del documento.

La llegada de los medios sociales ha originado la puesta en marcha de instrumentos que recogen, analizan y calculan, en tiempo real, el impacto de las menciones y comentarios online sobre la literatura científica y académica de un repositorio.

Estos instrumentos son conocidos como métricas alternativas o altmétricas y presentan una correlación con los índices de impacto basados en citas, pudiéndose emplear de una forma complementaria.

Diversas plataformas están al servicio de los repositorios, como Almetric.com, ImpactStory, Plos Altmetrics, Altmetric Explorer o PlumX, siendo en general de fácil instalación a través de las API de cada una de ellas. Hoy día, la herramienta más empleada y que más credibilidad ofrece es el índice Altmetric. Este monitorea varias redes sociales, entre las que destacan YouTube, Google, Reddit, Twitter, Facebook, Mendeley, ResearchGate, Reddit, LinkedIn, blogs, noticias, etc. Las menciones, 

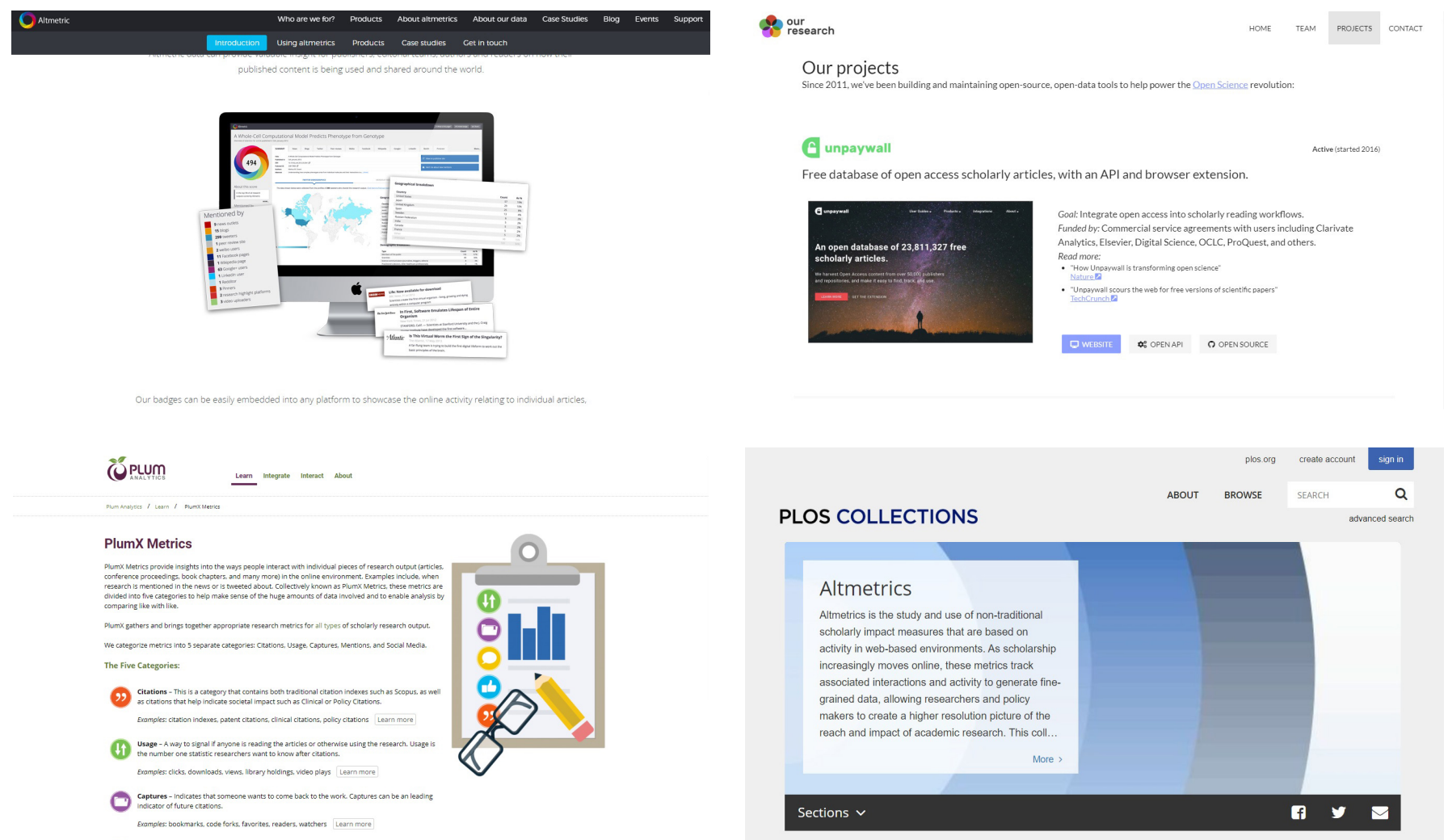

Las estadísticas de uso son esenciales, tanto para la institución académica como para el propio autor, al ofrecer la popularidad de un documento y su potencial de lectura y, en definitiva, el valor o influencia científica y académica del documento

visitas, descargas, comentarios y difusiones, entre otras acciones, generan un indicador numérico que aumenta cada vez que el soporte documental aparece en algunos de los medios indicados, despertando un cierto interés y aceptación.

Es importante darse cuenta de que el éxito de un repositorio se puede medir no solo a través de estadísticas, sino a partir del desarrollo de relaciones entre los bibliotecarios de disciplina, el equipo de comunicaciones, los centros, las escuelas y los institutos de investigación y con los académicos e investigadores.

Las herramientas digitales se encuentran en todos los procesos de la investigación; los nuevos paradigmas como el acceso abierto, métricas alternativas y redes sociales son un importante ejemplo de cómo estos cam- bios están afectando a la forma en que los investigadores se imaginan el futuro de las publicaciones académicas (ALONSO-ARÉVALO; CORDÓN-GARCÍA; MALTRÁS BARBA, 2016).

El valor de las métricas alternativas es más alto de lo que se piensa y se podría concentrar en tres grupos diferentes: autores, administradores del repositorio y gestores de la universidad.

$>$ Los autores, como suplemento del factor de impacto de una revista, pueden obtener las menciones (de medios sociales) para mejorar sus currículums. Por otro lado, pueden acceder a características demográficas e institucionales de quienes acceden a sus documentos y de esta forma se podría trazar una red profesional. 
> Los administradores de los repositorios se benefician del uso de métricas alternativas porque permiten incentivar y motivar a los autores para que el contenido sea abiertamente accesible, al ofrecerles más datos sobre el conocimiento del autor y las lecturas de su autoría, que podrían traducirse en citas bibliográficas. Además, es patente un aumento de la visibilidad y difusión de proyectos de investigación y sus consiguientes publicaciones de los resultados, fin deseado por los financiadores. Los medios sociales permiten construir una plena comunidad científica y académica en donde la imagen y las relaciones juegan un gran papel. Por último, las métricas alternativas pueden ayudar a planificar el desarrollo de la colección, los recursos y las actividades de marketing y divulgación de los contenidos bibliográficos de los repositorios.

> Por último, los gestores universitarios pueden emplear las métricas alternativas de cara a las políticas educativas, captación de estudiantes y reclutamiento de docentes e investigadores. Así mismo, los números que arrojan estas métricas pueden ser empleados por diferentes comisiones y comités: de selección, calidad, revisiones, etc. (KONKTIEL; SCHERER, 2013).

Como se ha demostrado, el uso de las métricas alternativas en los repositorios presenta claros beneficios pero, a partir de aquí, son las instituciones las que deben analizar la puesta en marcha e implementación de esta tipología de métricas.

La novedad del complemento implica el análisis de varios factores: elección de la herramienta, costes, normalización de la plataforma, seguimiento y el interés del usuario, fundamentalmente.

\section{BIBLIOGRAFÍA}

- ALONSO-ARÉVALO, J.; CORDÓN-GARCÍA, J. A.; MALTRÁS BARBA, B. (2016) Altmetrics: medición de la influencia de los medios en el impacto social de la investigación. Cuadernos de Documentación Multimedia [en línea], vol. 27 n. ${ }^{\circ}$ 1, $2016<$ https://revistas.ucm.es/index.php/CDMU/article/ view/52870> [Consulta: 07/05/2020]

- GONZÁLEZ-PÉREZ, L. I.; RAMÍREZ-MONTOYA, M. S.; GARCÍA-PEÑALVO, F. J. (2020) User experience in institutional repositories: A systematic literature review. En Digital Libraries and Institutional Repositories: Breakthroughs in Research and Practice. USA: Information Resources Management Association, 2020, p. 423-440

- KONKTIEL, S.; SCHERER, D (2013) New opportunities for repositories in the age of altmetrics. Bulletin of the American Society for Information Science and Technology [en línea], vol. 39, n. ${ }^{\circ} 4,2013$, pp. 22-26 <https://dl.acm.org/doi/abs/10.1002/ bult.2013.1720390408> [Consulta: 07/05/2020] 